\title{
El centro de investigación y capacitación en administración pública de costa rica como productor de conocimiento y tendencias científicas
}

\section{The Center for Research and Training in Public Administration of Costa Rica as a producer of knowledge and scientific trends}

\section{Montero Corrales, Carlos Alberto; Spatti, Ana Carolina}

\section{Carlos Alberto Montero Corrales}

Universidad de Costa Rica, Costa Rica Ana Carolina Spatti

Universidad Estatal de Campinas, Brasil

\author{
Documentos y Aportes en Administración Pública y \\ Gestión Estatal \\ Universidad Nacional del Litoral, Argentina \\ ISSN: 1666-4124 \\ ISSN-e: 1851-3727 \\ Periodicidad: Semestral \\ vol. 21, núm. 36, 2021 \\ Editor: José Vigil | jvigil@fce.unl.edu.ar \\ Recepción: 19 Abril 2021 \\ Aprobación: 15 Julio 2021
}

URL: https://doi.org/10.14409/daapge.2021.36.e0010 NoComercial-CompartirIgual 4.0 Internacional.

Para citar este artículo: Montero Corrales, C. A.; Spatti, A. C. (2021) "El Centro de Investigación y Capacitación en Administración Pública de Costa Rica como productor de conocimiento y tendencias científicas" DAAPGE Vol. 21, $\mathrm{N}^{\circ} 36$, 2021, pp. 28-50. UNL, Santa Fe, Argentina.
Resumen: El Centro de Investigación y Capacitación en Administración Pública (CICAP) de la Universidad de Costa Rica (UCR) es una institución que por 49 años ha funcionado como vehículo de difusión del campo de conocimiento de la Administración Pública en Costa Rica. Dada su importancia en generar y difundir conocimiento para mejorar la capacidad de gestión de lo público, este artículo presenta una caracterización de la producción científica del Centro, fundamentando la discusión en dos preguntas: ¿Cómo se puede tomar decisiones basadas en evidencia para publicar y difundir a nivel internacional la producción científica en Administración Pública? Y ¿De qué manera la producción científica realizada en el CICAP resulta ser un insumo para la toma de decisiones con mayor impacto científico en las investigaciones en el campo? Para esto, se utiliza una metodología mixta basada en un estudio bibliométrico y entrevistas. Como resultado, fue posible identificar tanto la dinámica de producción de conocimiento del Centro, como potenciales líneas de investigación en el campo de la Administración Pública: Desarrollo Municipal, Administración Pública, Aduanas y Comercio Exterior y Gobierno Abierto. Por lo tanto, el artículo ofrece un aporte empírico al CICAP y un aporte teórico al campo de la Administración Pública.

Palabras clave: bibliometría, planificación, evaluación, producción científica, Administración Pública.

Abstract: The Center for Research and Training in Public Administration (CICAP) of the University of Costa Rica (UCR) is an institution that, for 49 years, functioned as a vebicle for the diffusion of the field of knowledge of Public Administration in the country. Given its importance in generating and disseminating knowledge to improve public management capacity, we present a characterization of the scientific production of the Center, basing the discussion on two questions: How can evidence-based decisions be made to publish and disseminate scientific production in Public Administration at an international level? In what way does CICAP's scientific production results in input for decision-making with greater scientific impact on this field research? For this, we employ a mixed methodology based on a bibliometric study and 
switch. As a result, it is possible to identify both the dynamics of the production of knowledge of the CICAP, as well as potential lines of research in the field of Public Administration: Municipal Development, Public Administration, Customs and Foreign Trade and Open Government. Therefore, the article offers an empirical contribution to CICAP and a theoretical contribution to the field of Public Administration.

Keywords: bibliometric, planning, evaluation, scientific production, Public Administration.

\section{Introducción}

En el Plan Nacional de Ciencia, Tecnología e Innovación (2015-2021) de Costa Rica, se menciona que la plataforma actual para la ciencia endógena es frágil, poco o mal incentivada y relativamente desconocida. La inversión en $\mathrm{I}+\mathrm{D}$ (Investigación y Desarrollo) es menor al esperado según el nivel de desarrollo del país (0.9\% del PIB, esto al año 2014). En este sentido, se hace urgente promover la investigación en torno a temas relevantes para el desarrollo nacional, desde una perspectiva multidisciplinaria e interinstitucional (MICITT, 2019).

En esta misma línea, se discute teóricamente que los Centros de investigación planifiquen estratégicamente su actividad científica, ya que el desarrollo científico, tecnológico y de innovación compone la base fundamental de valor agregado en la sociedad contemporánea; en la medida en que las organizaciones que producen conocimiento y tecnologías interfieren los procesos sociales y la creación y distribución de la renta, la competitividad y el desarrollo de los países (Bin, 2008; Furtado et. al, 2009; Cozzens, 2012; Benavente et al., 2012; Lin et al., 2014). Es por esta razón que investigar en el campo de la Administración Pública es esencial para fortalecer la fragilidad de la ciencia producida en Costa Rica.

En este mismo sentido, varias personas autoras han utilizado el análisis bibliométrico para caracterizar la producción científica en Administración Pública. Sin embargo, lo han realizado con recortes específicos en áreas temáticas o alcance del análisis: Aguilera Gámez (2020) se interesó en el tema da transformación digital en el sector público; Cicea (2020) se centró en performance; Juliani \& Oliveira (2016) en gestión del servicio público; Hocayenda-Silva, Rossoni \& Ferreira Júnior (2008) en gestión social; Dias, Zarelli \& Selig (2014) en capital intelectual; y Fadul (2011) enfoca en el análisis de Anais brasileños. Por lo tanto, conforme afirma Pérez (2007), faltan instrumentos e indicadores para cuantificar la difusión y producción de las publicaciones en el campo de la Administración Pública, lo cual revista la pertinencia teórica y metodológica de este estudio.

Los datos de Scival Platform (una solución de Elsevier basada en registros de publicaciones de Scopus) revelan que la producción científica en el mundo sobre Administración Pública se ha centrado especialmente en los siguientes temas: "Motivación del servicio público", "Gobierno abierto" y "Gobernanza de la red" (Cuadro 1). Estos tres temas tienen una alta "prominencia" (prominence), ${ }^{2}$ variable que mide el momentum/protagonismo de un tema de investigación 
en particular (indicando cuáles están atrayendo más atención por parte de investigadores e instituciones), variando, cada vez más, entre 0 y 100.

\section{Cuadro 1.}

Publicaciones en administración pública en el mundo por tema (top 10) y percentil de prominencia (2018-2020)

\begin{tabular}{lll}
\hline Tema & $\begin{array}{l}\text { Numero de publicaciones en Administración } \\
\text { Pública }\end{array}$ & Percentil de prominencia \\
\hline Motivación en el Servicio Público; Motivación; Red Tape; Funcionarios públicos & 421 & 97.9 \\
Gobierno Abierto; Participación electrónica; Gobernanza electrónica & 373 & 99.5 \\
Gobernanza en red; colaboración interorganizacional; Respuesta a desastres & 350 & 98.2 \\
Estado de bienestar; inversión social; Politicas de Mercado de trabajo & 218 & 97.8 \\
New Public Management; Agencias regulatorias; responsabilidad social & 192 & 93.9 \\
Corrupción; sobornos, medidas de anti-corrupión & 175 & 98.7 \\
Accrual Accounting; International Public Sector Accounting Standards (IPSAS) & 152 & 94.8 \\
Acto de aire limpio; National Ambient Air Quality Standards; Aprobación & 51.3 \\
Sistemas socio-ecológicos; resiliencia ecológica; marco de referencia acerca la coalición de abogacia & 140 \\
Anti lavado de dinero; financiamiento del terrorismo; crímenes financieros & 141 & 99.7 \\
\hline
\end{tabular}

Scival (Elsevier).

En relación a Costa Rica, según datos de la Plataforma Scival, la producción científica del país en la misma área de conocimiento involucra, predominantemente, los temas relacionados a: "gobierno abierto", "sistema de pensión", y "política pública".

Así, considerando la importancia de los centros de investigación como vehículo de comunicación y difusión del desarrollo de la Administración Pública, el presente artículo tuvo el objetivo de caracterizar la producción científica de los últimos 20 años del Centro de Investigación y Capacitación en Administración Pública (CICAP), a fin de generar conocimiento para la toma de decisiones en líneas de investigación estratégicas para el Centro.

Ubicado en la Universidad de Costa Rica (UCR), el CICAP fue fundado en 1972 con el objetivo de formar y actualizar a los cuadros profesionales de la administración pública costarricense, así como de investigar las principales problemáticas que aquejaba la expansión del Estado costarricense en aquellos años (Moya, 2012). En este sentido, por producir y difundir conocimiento para mejorar la capacidad de gestión de lo público, mediante la investigación y la acción social, es una institución importante en el campo científico de la Administración Pública. Así mismo, el CICAP pertenece al área científica y tecnológica de las Ciencias Sociales, la cual durante el año 2018 ocupó la primera posición de inversión en Investigación y Desarrollo en Costa Rica, con un 26\% sobre el total invertido en el país (MICITT, 2019).

Por lo anterior, esta investigación tuvo como eje probletromatizador la siguiente pregunta: ¿De qué manera la producción científica realizada en el CICAP durante el período 2010-2020 es un insumo para la toma de decisiones con mayor impacto científico en las investigaciones en el campo de la administración pública?

Para responder a esta pregunta, se alcanzaron los siguientes objetivos específicos:

1. Identificar las áreas temáticas de la Administración Pública en las cuales se han producido resultados de investigación, por medio de 
la construcción participativa con grupos de investigación afines al campo;

2. Visualizar los resultados de búsqueda del estudio bibliométrico a fin de identificar áreas temáticas y líneas de investigación estratégicas para el CICAP, y

3. Destacar los intereses de cada grupo de investigación y los resultados de búsqueda para orientar la producción científica del CICAP y del campo de Administración Pública.

A continuación, se presentará la metodología, los resultados, las discusiones respectivas y las consideraciones finales.

\section{Metodología}

La metodología tuvo como punto de partida teórico la definición realizada por Price (1963) de Ciencia, la cual es considerada como aquello que se edita en las publicaciones científicas y cuya evidencia son documentos científicos públicos. A pesar de que la definición es bastante general, la misma permite medir la actividad científica tomando en consideración las características de las publicaciones; lo cual, para Price, es la manera de conectar la investigación con la comunidad científica.

A partir de este concepto de Ciencia, se desprende el concepto de bibliometría, basado en el cálculo y en el análisis de los resultados de búsqueda de lo que es cuantificable en la producción y en el consumo de la información científica (Spinak 1996).

Por lo tanto, este artículo parte de que la bibliometría es una herramienta matemática y estadística para mapear índices de producción científica (Araújo, 2006), o bien para medir la Ciencia desde la definición de Price (1963), contribuyendo para explorar, organizar y analizar grandes cantidades de datos. $\mathrm{Al}$ hacer un "mapeo científico" (Chandra \& Walker, 2019), el instrumento permite la organización y sistematización de la información científica y tecnológica, la generación de indicadores para el tratamiento y manejo de la información y el abordaje de problemas para futuras investigaciones (Chueke \& Amatucci, 2015). En ese sentido, la bibliometría ayuda a las personas investigadoras en la toma de decisiones (Daim et al., 2006) y en la evaluación del desempeño técnico-científico de una organización, región o de una nación (Freitas, 1997).

Dicho lo anterior, la investigación se fundamentó en la siguiente pregunta: ¿Cómo se puede tomar decisiones basadas en evidencia para publicar y difundir a nivel internacional la producción científica del CICAP de la Universidad de Costa Rica?

Inicialmente, esta pregunta se responde con la siguiente hipótesis: la producción científica del Centro en los últimos 20 años es un referente para tomar decisiones basadas en evidencia hacia el futuro, tomando en consideración la producción científica mundial en áreas temáticas prioritarias para el Centro y de relevancia para el área de conocimiento de la Administración Pública.

A fin de proponer una metodología acorde con estas premisas teórico metodológicas, se explicarán las cuatro etapas que se siguieron en la investigación. La primera etapa se basó en la revisión documental de resultados de investigación 
del CICAP en los últimos 20 años, tomando como alcance temporal el recomendado por Chueke y Amatucci (2015), quienes defienden un período mínimo de 15 o 20 años para captar la evolución de áreas temáticas. La revisión documental recabó información a partir de las siguientes fuentes de información presentados en el Cuadro 2:

\section{Cuadro 2}

Fuentes de información y alcance temporal de los medios de información para la revisión documental*

\begin{tabular}{|l|l|}
\hline Medios de información & Período \\
\hline $\begin{array}{l}\text { Página web del CICAP Sobre reporte de } \\
\text { investigaciones }\end{array}$ & $\begin{array}{l}\text { No se } \\
\text { aplica }\end{array}$ \\
\hline $\begin{array}{l}\text { Reporte histórico del Sistema de Información y } \\
\text { Gestión de Programas, proyectos y actividades } \\
\text { (SIGPRO) }\end{array}$ & $2000-2020$ \\
\hline $\begin{array}{l}\text { Reporte del programa ED 2577 Programa de Asesoria } \\
\text { Técnica y Consultoría, revisado en el sistema de } \\
\text { información institucional de la UCR: Bite }\end{array}$ & $2000-2020$ \\
\hline $\begin{array}{l}\text { Reporte del Sistema de Información de Unidades de } \\
\text { Investigación (SIUDI). }\end{array}$ & $2000-2020$ \\
\hline $\begin{array}{l}\text { *Programa 3159 Programa de Desarrollo Municipal, } \\
\text { revisado en el sistema de información institucional } \\
\text { de la UCR: Bite. }\end{array}$ & $2015-2020$ \\
\hline $\begin{array}{l}\text { **Reporte de Productividad Académica Estancia } \\
\text { Posdoctoral del Dr. Rodolfo Martínez. }\end{array}$ & $2012-2016$ \\
\hline $\begin{array}{l}\text { ***Programa 2578 Capacitación del Archivo } \\
\text { UniverSitario Rafael Obregón Loría. }\end{array}$ & $\begin{array}{l}2009- \\
2012\end{array}$ \\
\hline
\end{tabular}

Fuente: elaboración propia.

${ }^{*}$ No hay reportes anteriores al año 2015 en el sistema de información Bite de la UCR.

** El Reporte de Productividad Académica Estancia Posdoctoral empezó en el año 2012.

*** No hay reportes anteriores al año 2009 en el Programa 2578 de la UCR.

Como resultado, se identificaron 145 documentos científicos generados en el Centro durante el periodo 2000-2020, incluyendo proyectos de investigación, artículos científicos, capítulos de libros, libros, manuales, guías, informes de investigación, seminarios, congresos, y foros.

Posteriormente, a partir de la técnica de criterio experto, se asignó a cada uno de los 145 documentos científicos una área temática clasificada por Smolki et al. (2017) en la Administración Pública, logrando descubrir cuales habían producido más resultados científicos en los últimos 20 años, y destacando cuatro áreas en las que el CICAP ha generado mayor cantidad de resultados, a saber: Administración Pública con 19 resultados, Desarrollo Municipal con 17, Gobierno Abierto con 17 y Administración Aduanera con 16 resultados.

Una vez identificadas estas áreas temáticas, para definir una línea de investigación, se procedió a la segunda etapa de la investigación: realización de entrevistas semiestructuradas (marzo de 2020) y sesiones de trabajo (julio de 2020) con las personas investigadoras de cada una de las cuatro áreas temáticas mencionadas y con la persona coordinadora del área de investigación y desarrollo del Centro. Así, fueron entrevistadas un total de ocho personas, siendo siete investigadoras en las cuatro áreas y una persona con funciones de coordinación de la producción científica. Las preguntas orientadoras de las entrevistas fueron las siguientes: 
- ¿Cuáles proyectos de investigación se desarrollan en esta materia o se pretenden desarrollar?

- ¿Cuál considera que es el logro en investigación más significativo que ha tenido el laboratorio en los últimos años?

- ¿Cómo se caracteriza la participación en redes de investigadores en la materia?

- ¿Cuál las líneas de investigación más relevantes para que el CICAP desarrolladas en los próximos 6 años?

- ¿Cuáles considera que son las palabras clave que caracterizan esta línea?

- ¿Cuáles palabras clave considera que deben ser excluidos de la búsqueda?

- ¿Qué información adicional sobre la producción científica de la línea de investigación priorizada és relevante para tomar decisiones estratégicas sobre las actividades del Centro?

En el estudio, se consideró una línea de investigación como el proceso de investigación contemporáneo, colectivo y articulado institucionalmente, que realiza un trazo imaginario que permite determinación de la orientación teória y del establecimiento de procedimientos metodológicos, o de que será investigado en un contexto o realidad; delimitando las fronteras del campo de conocimiento específico en que está inserta la investigación (Menandro, 2003). En cada una de las líneas de investigación, se realizó un estudio bibliométrico a fin de trazar la trayectoria de la producción científica identificada en Scopus Elsevier. Dichas áreas temáticas fueron consideradas por la Dirección del Centro como estratégicas en el marco de la planificación a mediano plazo de la Unidad Académica, definiendo así las siguientes líneas de investigación:

a) Rol de la administración pública en el desarrollo de la gestión del territorio en el marco del capitalismo,

b) Gestión del territorio y territorialidad,

c) Instrumentos de medición de la transparencia y participación en el sector público y

d) Sistemas informáticos aduaneros y facilitación del comercio.

En las entrevistas realizadas, las anteriores líneas de investigación se justificaron como estratégicas durante los 20 años en estudio por las siguientes razones: el CICAP ha invertido recursos en estas líneas de investigación en el período de estudio; en los últimos 20 años existen personas investigadoras del Centro estudiando temas en las cuatro líneas de investigación y publicando en revistas indexadas nacionales, y finalmente las líneas de investigación se consideraron como necesarias de investigar para aportar al desarrollo de la administración pública costarricense (Persona entrevistada 1 y 2, comunicación personal, 22 de Junio y 17 de marzo de 2020).

Con los resultados generados en las entrevistas, y tomando en consideración las palabras claves recabadas, se construyeron las ecuaciones de búsqueda expresadas en el Cuadro 3, las cuales se ejecutaron en la base de datos en Scopus para generar una base de datos de la producción científica mundial en cada línea de investigación: 


\section{Cuadro 3.}

Ecuaciones de búsqueda por área temática prioritaria del CICAP

\begin{tabular}{|c|c|c|}
\hline $\begin{array}{l}\text { Área temática } \\
\text { estratégica del } \\
\text { CICAP }\end{array}$ & \begin{tabular}{|l|} 
Línea de \\
investigación \\
identificada
\end{tabular} & Ecuación de búsqueda usada en Scopus \\
\hline $\begin{array}{l}\text { Administración } \\
\text { pública. }\end{array}$ & $\begin{array}{l}\text { Rol de la } \\
\text { administración } \\
\text { pública en el } \\
\text { desarrollo de } \\
\text { la gestión del } \\
\text { territorio en el } \\
\text { marco del } \\
\text { capitalismo. }\end{array}$ & $\begin{array}{l}\text { State AND government AND capitalism AND "public administration" AND (theory OR approach } \\
\text { ) AND evolution AND ("economic development" OR "human development" OR "Sustainable } \\
\text { development") AND sector AND NOT environment AND NOT arts AND NOT humanities }\end{array}$ \\
\hline $\begin{array}{l}\text { Desarrollo } \\
\text { Municipal }\end{array}$ & $\begin{array}{l}\text { Gestión del } \\
\text { territorio y } \\
\text { territorialidad }\end{array}$ & $\begin{array}{l}\text { globalization AND territoriality AND ("institutional change" OR urbanization OR "public } \\
\text { land") AND development AND NOT geology AND NOT farm AND NOT arts }\end{array}$ \\
\hline $\begin{array}{l}\text { Gobierno } \\
\text { Abierto }\end{array}$ & $\begin{array}{l}\text { Instrumentos } \\
\text { de medición } \\
\text { de la } \\
\text { transparencia } \\
\text { y } \\
\text { participación } \\
\text { en el sector } \\
\text { público }\end{array}$ & $\begin{array}{l}\text { "Open data" } \\
\text { AND (index OR evaluation OR assessment) AND transparency AND accountability AND ("citizen } \\
\text { participation" OR "public participation") OR Collaboration OR cocreation AND NOT e- } \\
\text { government AND NOT "digital government" AND NOT "Social peace" AND NOT human }\end{array}$ \\
\hline $\begin{array}{l}\text { Administración } \\
\text { aduanera }\end{array}$ & $\begin{array}{l}\text { Sistemas } \\
\text { informáticos } \\
\text { aduaneros y } \\
\text { facilitación } \\
\text { del comercio. }\end{array}$ & $\begin{array}{l}\text { Commerce OR custom AND (control OR fiscalization) AND "economy integration" OR "trade } \\
\text { facilitation" OR "System integration" OR "Custom law" AND NOT "foreign Trade" AND } \\
\text { NOT "international trade" AND NOT energy AND NOT electronic AND NOT industrial AND } \\
\text { NOT migration AND NOT human AND NOT population AND NOT web AND services AND } \\
\text { NOT design AND NOT economics AND NOT circuits }\end{array}$ \\
\hline
\end{tabular}

Fuente: elaboración propia según entrevistas realizadas.

La base de datos Scopus Elsevier's fue seleccionada por permitir la consulta y el acceso a las referencias bibliográficas en diferentes campos del conocimiento, entre ellas las Ciencias Sociales. Según Mongeon y Hus (2016), Scopus almacena 5682 revistas $(27,8 \%$ del total de revistas indexadas en Scopus) en el área del conocimiento en Ciencias Sociales, entre las cuales en el campo de la Administración Pública se encuentran las siguientes: Public Administration Review, Australian Journal of Public Administration, Public Administration, Canadian Public Administration, International Journal of Public Administration y International Review of Administrative Sciences, entre otras.

En la tercera etapa de la metodología, se tomó cada una de las cuatro bases de datos resultantes de la aplicación de la ecuación de búsqueda en Scopus y se analizaron según las preguntas:

- ¿Cuál es el comportamiento de la producción científica indexada en Scopus en la línea de investigación priorizada por el Centro?

- ¿Quiénes son las diez primeras personas autoras que registran la mayor cantidad de publicaciones indexadas en Scopus en esta línea de investigación?

- ¿De qué manera se relacionan los diez primeros países en los que se publica más producción científica en esta línea de investigación?

- ¿En qué medios se difunde la mayor cantidad de producción científica indexada en Scopus en la línea de investigación priorizada?

Para finalizar, en la cuarta etapa se visualizaron estadísticamente los resultados con la finalidad de llegar a debatir acerca de los productos de investigación que aportan al crecimiento y maduración de la investigación en Administración Pública y acerca de la necesidad de tomar decisiones basadas en evidencia para generar iniciativas no aisladas de generación de conocimiento en el CICAP. 


\section{Resultados}

Los resultados del presente artículo se calcularon tomando en consideración las bases de datos explicadas en la sección de metodología, una correspondiente a los 145 documentos científicos del Centro y cuatro bases correspondientes a la búsqueda bibliométrica realizada en Scopus, esto a fin de calcular la producción científica del CICAP en los últimos 20 años y observar el estado de situación del conocimiento en las áreas temáticas priorizadas y sus líneas de investigación a nivel mundial. Ambos resultados se muestran a continuación.

\subsection{Resultados bibliométricos de la producción cientifica del CICAP}

En cuanto a la base de datos de la producción científica del CICAP, se identificaron un total de 145 resultados científicos distribuidos entre: inscripción de proyectos de investigación ante la Vicerrectoría de Investigación, publicación de artículos científicos en revistas indexadas o en espacios institucionales de la UCR, publicación de capítulos de libros y libros, publicación de memorias, guías y manuales, y la organización de congresos, seminarios y foros.

Según lo anterior, cabe preguntarse acerca de la trayectoria de la producción científica del CICAP en los últimos 20 años a fin de elucidar el comportamiento en el tiempo de los 145 resultados mencionados. Para este fin, se muestra el Gráfico 1:

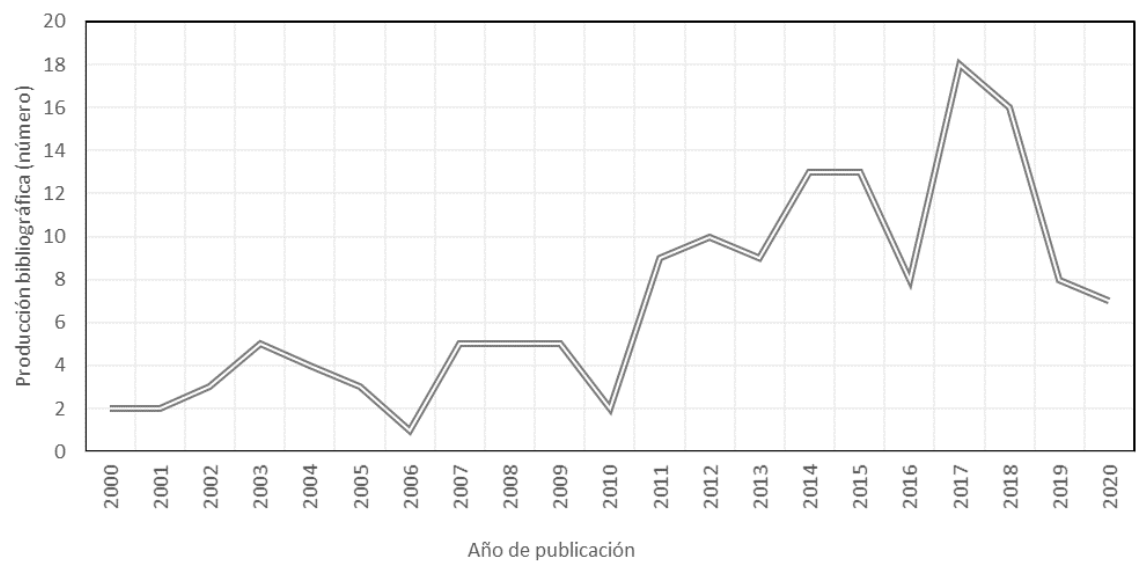

Gráfico 1.

Producción bibliográfica del CICAP, en números absolutos (2000-2020)

Fuente: elaboración propia.

Es evidente que durante el año 2000 al año 2010 la producción científica del CICAP es bastante escasa, siendo que durante estos 10 años se lograron 37 resultados científicos, teniendo mayor logro la organización de congresos, seminarios, congresos y simposios. Es a partir del año 2010 que se denota un cambio sustantivo al alza para aumentar significativamente la producción científica en el Centro, lo cual se explica debido a la contratación de una persona en tiempo completo para realizar labores de investigación y coordinación de las acciones de investigación y desarrollo del CICAP (Persona entrevistada 1, comunicación personal, 22 de Junio de 2020). ${ }^{3}$ El año 2010 marca un antes y un después en la producción científica del CICAP, ya que este fue el 
punto de inflexión en el que se deja de darle prioridad a la organización de congresos, seminarios y foros, y se le otoga mayor prioridad a la publicación como resultados de la investigación realizada en el Centro (Persona entrevistada 1 y 7 , comunicación personal, 22 de junio y 17 de marzo de 2020).

Los 145 resultados recopilados en la base de datos de la producción científica durante los años 2000-2020 se distribuyen de la siguiente manera según tipo de producción científica (Gráfico 2):

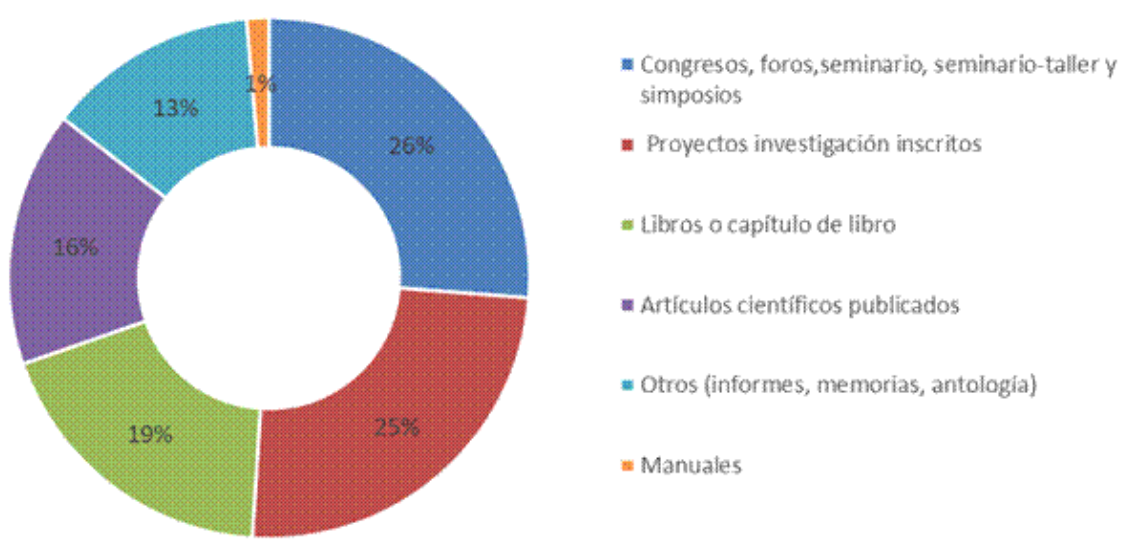

Gráfico 2 .

Distribución por tipo de producción científica del CICAP (2000-2020) Fuente: elaboración propia.

Tal cual se muestra en el Gráfico 2, la organización de congresos, seminarios, foros y simposios representa un $26 \%$, siendo un total de 38 eventos organizados en el período. Por su parte, los proyectos de investigación inscritos en la Vicerrectoría de Investigación de la Universidad de Costa Rica representan un $26 \%$ también, lo cual equivale a 36 proyectos de investigación.

En cuanto a la publicación de capítulos de libros y libros, dicho tipo de producción científica representa un 19\%, lo cual equivale a 27 resultados. Por su parte, la publicación de artículos científicos representa un valor de $16 \%$, teniendo como resultado un total de 23 publicaciones. Finalmente, en la categoría de Otros, se registran 19 resultados; y Manuales se registran 2 resultados.

Estos datos muestran que ha existido una alta vocación del Centro dirigida a la organización de congresos, seminarios, foros y simposios, mientras que las actividades de publicación de documentos científicos no han tenido el mismo comportamiento en el tiempo. En este sentido, cabe preguntarse ¿qué tipo de producción científica se ha alcanzado a partir del año 2010 debido al aumento considerable de la publicación científica en el Centro?. Para responder esta inquietud, se muestra el Gráfico 3, con la trayectoria anual por tipo de producción científica (proyectos de investigación, artículos indexados publicados y capítulos de libros y libros publicados): 


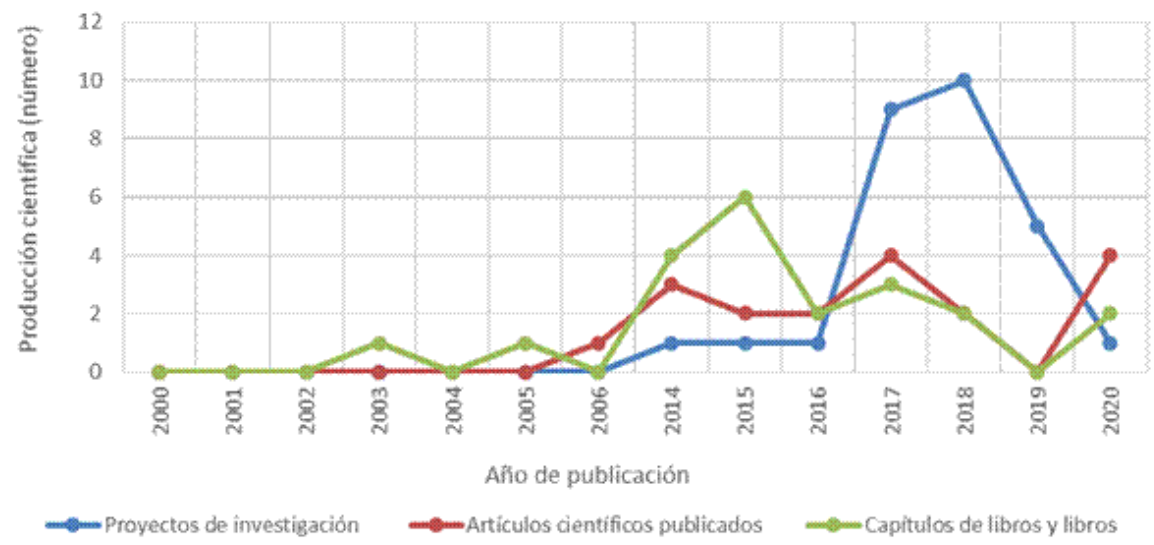

Gráfico 3.

Trayectoria anual por tipo de producción científica del CICAP (2000-2020) Fuente: elaboración propia

El Gráfico 3 evidencia un aumento en la inscripción de proyectos de investigación, de la publicación de artículos y de la publicación de capítulos de libros y de libros, sin embargo es evidente que a partir del año 2017 hasta el año 2019 existe una caída acelerada de la producción científica. Es relevante mencionar que de los 145 resultados científicos recabados en la base de datos, 90 se distribuyeron en los tres anteriores tipos de producción científica, correspondientes a proyectos de investigación, publicación de artículos y capítulos de libros y libros.

Por otra parte, la organización de congresos, seminarios, foros y la publicación de otros (informes, manuales y guías) presenta un comportamiento constante desde el año 2000 a la actualidad, dicho rubro no sufrió un aumento acelerado a partir del año 2010, exceptuando la organización de congresos, seminarios y foros la cual desde el año 2017 se encuentra en 0 resultados. Dicho comportamiento se muestra a continuación (Gráfico 4):

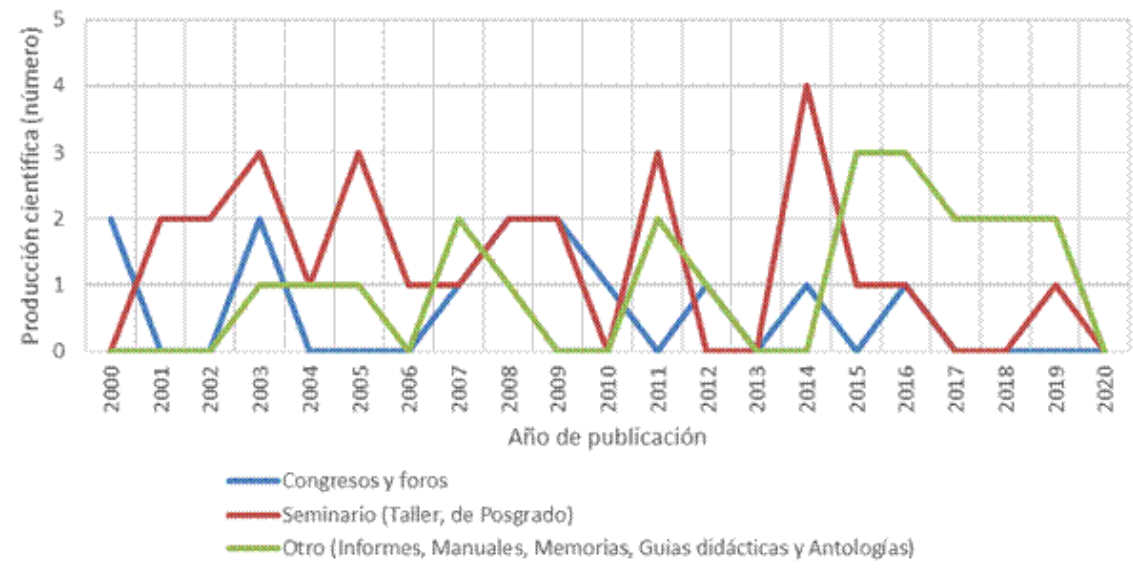

Gráfico 4.

Trayectoria anual por tipo de producción científica del CICAP (2000-2020) Fuente: elaboración propia

El Gráfico 4 evidencia que dichos tipos de producción científica no tuvieron un aumento acelerado en el período de estudio, por el contrario, la organización de Congresos y Seminarios tiende a la baja a partir del año 2014, lo cual llama la 
atención, ya que, antes de ese año, este tipo de producción científica era la que poseía mayor frecuencia de resultados en este estudio.

\subsection{Resultados bibliométricos de las cuatro áreas temáticas}

En cuanto a las áreas temáticas, el Centro ha investigado en diversos campos a lo largo de los años. Específicamente tomando como referencia las áreas temáticas de la Administración Pública propuestas por Smolski et al. (2017), el CICAP ha realizado alguna investigación o publicación en la materia en treinta y nueve ${ }^{4}$ de ellas. Según lo anterior, cabe preguntarse en ¿Cuáles de éstas áreas temáticas existe mayor producción científica en los últimos años 20 años en el Centro? Para contestar lo anterior, se seleccionaron las primeras 10 áreas temáticas con mayor cantidad de resultados científicos, los cuales se muestran en el siguiente Gráfico 5:

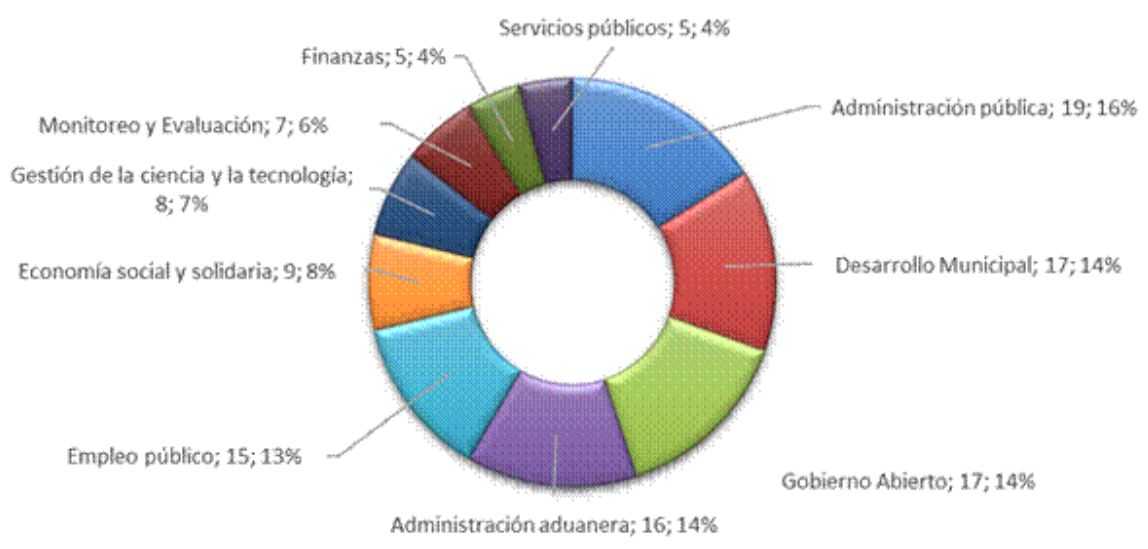

Gráfico 5.

Producción científica del CICAP distribuida en las 10 primeras áreas temáticas (2000-2020) Fuente: elaboración propia.

Según el Gráfico 5, las cuatro primeras áreas temáticas en las que se ha producido mayoritariamente resultados científicos son las siguientes: Administración Pública, Desarrollo Municipal, Gobierno Abierto y Administración Aduanera, de las cuales, exceptuando el área de Gobierno Abierto, son áreas temáticas que han caracterizado los resultados científicos del Centro desde su existencia.

El área temática correspondiente a Gobierno Abierto se considera como un área temática emergente debido a que desde el año 2015 se publica el informe de investigación acerca del Índice de Transparencia del Sector Público (2015, 2016, 2017, 2018, 2019, 2020), a través del patrocinio de la Defensoría de los Habitantes de la República de Costa Rica, lo cual llama bastante la atención como un resultado con potencial de publicarse en artículos científicos o en su defecto en un libro. Esto se refuerza según datos de la Plataforma Scival, en la cual Gobierno Abierto es un tema asociado a un indicador de prominencia alto.

A continuación, se mostrarán los resultados bibliométricos por cada una de las cuatro áreas temáticas y líneas de investigación priorizadas en el CICAP, a fin de identificar el panorama mundial para encontrar rutas de la difusión de la producción científica venidera del Centro. 


\section{Area temática: Administración Pública}

Durante el proceso de entrevistas, se identificó que la línea de investigación "Rol de la administración pública en el desarrollo de la gestión del territorio en el marco del capitalismo" sería un tema relevante para explorar por medio de la bibliometría (Persona entrevistada 2, comunicación personal, 17 de marzo del 2020), esto debido a que, según la persona entrevistada, ha habido producción científica destacada en el tema en diferentes latitudes del mundo, como en México, Australia, Reino Unido, Norteamerica, Canada, Chile, Colombia y Ecuador.

Al realizar la búsqueda en Scopus Elsevier y tomando en consideración la ecuación de búsqueda mencionada en la metodología, se generaron 261 resultados entre los cuales se identifican un $48 \%$ de libros, un $37 \%$ de artículos científicos, un $9 \%$ de capítulos de libro, un $4 \%$ de Revisiones, un $1 \%$ de papers en Conferencias y un $1 \%$ en Editoriales. Es decir, la comunidad de investigación al publicar acerca del área temática "Rol de la administración pública en el desarrollo de la gestión del territorio en el marco del capitalismo", mayoritariamente utiliza los medios de publicación de libros y de artículos científicos.

Acerca de la trayectoria al largo de los años, la primera publicación en la línea de investigación fue en 1981. Sin embargo, el Gráfico 6 enfoca en la dinámica científica para el período de análisis 2000-2020.

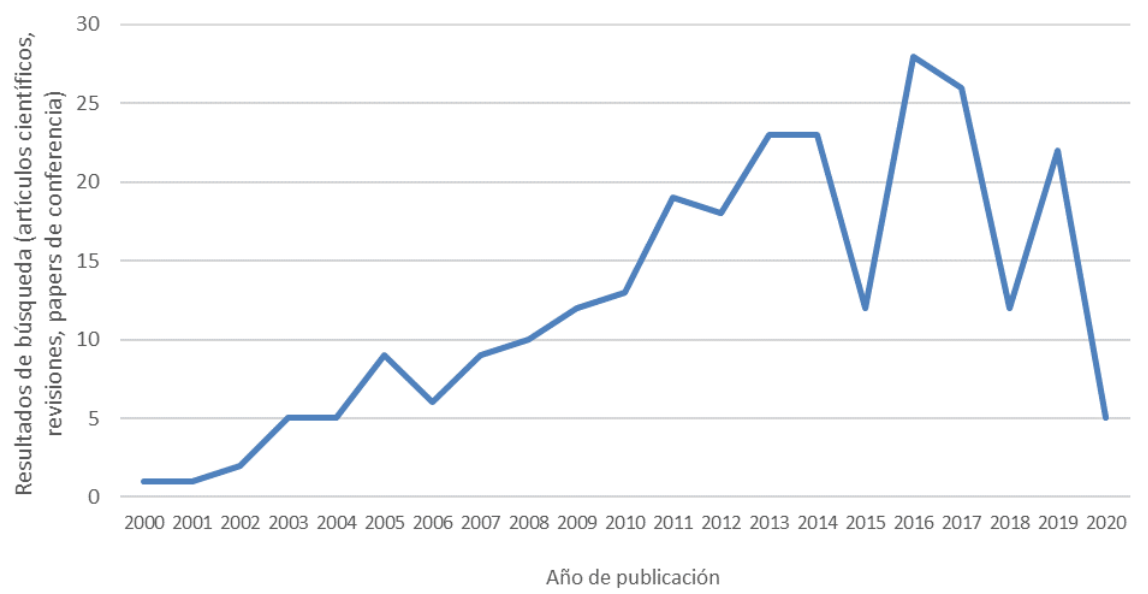

Gráfico 6.

Trayectoria de la producción científica. Línea de investigación: Rol de la administración pública en el desarrollo de la gestión del territorio en el marco del capitalismo (2000 - 2020)

Fuente: elaboración propia.

A partir del año 2005, se publicó una cantidad creciente de artículos científicos en esta materia, siendo que tan solo en el año 2014 se realizaron 23 publicaciones registradas en Scopus, siendo que a partir este año el comportamiento de la producción científica se torna inconstante con picos y saltos en la cantidad de publicaciones, pasando de 12 publicaciones en el 2015, 28 en el año 2016, 26 en el 2017, 12 en el 2018 y 22 en el 2019.

Por otro lado, en cuanto a la participación de personas autoras en la publicación de artículos, los resultados arrojan que existe una cantidad considerable de investigadores que publican más en esta línea de investigación, 
destacando a: Felock, R., Angelino, A., Angeir, H., Di Tomasso, Eom, T., Estevez, E., Forrest, T., Gal, J., Hooper, T. y Janowski, T.

Las personas autoras mencionadas publican mayoritariamente en revistas indexadas en el idioma inglés y registradas en Scopus, siendo las siguientes 10 revistas las que se concentran la mayor cantidad de publicaciones en la materia (Tabla 1):

\section{Tabla 1.}

Top 10 de las revistas en las que se publica más acerca de la línea de investigación Rol de la administración pública en el desarrollo de la gestión del territorio en el marco del capitalismo (2000 - 2020)

\begin{tabular}{|ll|}
\hline Nombre de la revista & $\begin{array}{l}\text { Número de } \\
\text { artículos } \\
\text { publicados }\end{array}$ \\
\hline $\begin{array}{l}\text { Development And Welfare Policy In South Asia } \\
\text { Annual Review Of Political Science }\end{array}$ & 5 \\
Chinese Business In Southeast Asia Contesting & 2 \\
Cultural Explanations Researching & 2 \\
Entrepreneurship & \\
Critical Perspectives On Accounting & 2 \\
Democracy Law And Governance & 2 \\
Digital Government Technology And Public & 2 \\
Sector Performance, & \\
International Journal of Public Administration & 2 \\
Law And Development Review & 2 \\
Policy Studies Journal & 2 \\
Political Economy Of Aid And Accountability & 2 \\
The Rise And Fall of Budget Support In & \\
Tanzania & \\
\hline
\end{tabular}

Fuente: elaboración propia

Por otro lado, en cuanto a las instituciones académicas que más han publicado en los últimos 10 años en esta materia, se destacan la Florida State University con 4 publicaciones registradas; y con 3 publicaciones se destacan el Business School University del Reino Unido, la University of Sussex y la Yonsei University. Con 2 publicaciones, se destacan el Departmen of Politics and Public Administration de la Universidad de Hong Kong y la City University London.

\section{Área temática: Desarrollo Municipal}

$\mathrm{Al}$ realizar la búsqueda en Scopus Elsevier tomando en consideración la ecuación de búsqueda mencionada en la metodología, se generaron 626 resultados de búsqueda entre los cuales se publica un $60 \%$ en formato artículo científico, un $24 \%$ en libros, un $11 \%$ de capítulso de libro, un $4 \%$ de Revisiones, un $1 \%$ en Editoriales. Es decir, la comunidad de investigación al publicar acerca del área temática de desarrollo municipal y en particular en la línea de investigación: "Gestión del territorio y territorialidad", mayoritariamente utiliza los medios de publicación de artículos científicos, libros y capítulos de libros. A continuación, se muestra la trayectoria de la producción científica de esta línea de investigación (Gráfico 7): 


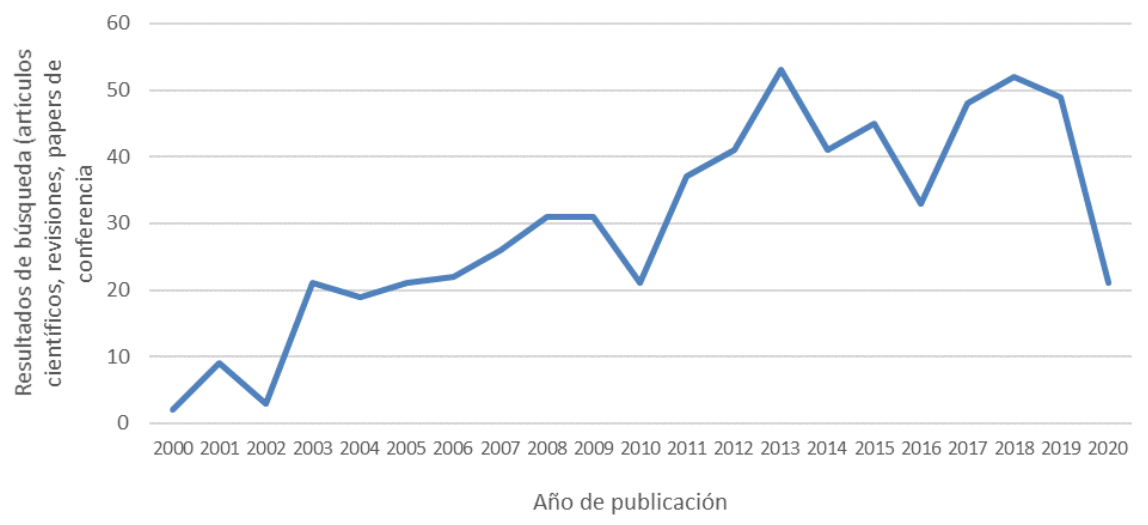

Gráfico 7.

Trayectoria de la producción científica. Línea de investigación:

Gestión del territorio y territorialidad (2000 - 2020).

Fuente: elaboración propia.

A diferencia del área temática de Administración Pública, esta línea de investigación inicia en el año 1994 con la primera publicación, lo cual muestra lo reciente de las producciones científicas en el campo. A partir del año 2000, la dinámica de publicación en estudio, prácticamente, se ha mantenido en un crecimiento constante, siendo el 2010, el 2014 y el año 2016, los años en los cuales se identifican disensos considerables de la publicación en la materia.

Los resultados también arrojan que existen autores cuya cantidad de publicaciones destacan por sobre el top 10 de autores que más han publicado en la materia: Brenner, N., que registra 9 publicaciones al año 2020; Jonas, A., que registra 7 publicaciones; Derudder, B., Jones, M. Jones, R., Meir, A. registran 6 publicaciones; Macleod, K. y Ward, K. registran 5 publicaciones; Addie, J. y Boelens, R. con 4 publicaciones; finalmente, con 2 publicaciones, se registra al autor Pike, A.

$\mathrm{Al}$ igual que en el área temática Administración Pública, las personas autoras en esta línea de investigación publican mayoritariamente en el idioma inglés, siendo las siguientes 10 revistas en las que se más publica en la materia (Tabla 2): 
Tabla 2.

Top 10 de las revistas en las que se publica más acerca de la línea de investigación. Gestión del territorio y territorialidad (2000 - 2020)

\begin{tabular}{|ll|}
\hline Nombre de la revista & $\begin{array}{l}\text { Número de } \\
\text { artículos } \\
\text { publicados }\end{array}$ \\
\hline $\begin{array}{l}\text { International Journal of Urban and Regional } \\
\text { Research }\end{array}$ & 18 \\
Environment and Planningy Progress in & 17 \\
Human Geography & \\
Political Geography & 14 \\
Urban Studies & 11 \\
Geoforum, Regional Studies, Territory Politics \\
Governance y Transactions of The Institute of \\
British Geographers
\end{tabular}

Fuente: elaboración propia

En cuanto a las instituciones académicas que han publicado en los últimos 10 años en esta materia, se destacan el University College of London con 6 publicaciones, el Department of Geography de la Universidad de Kentucky con 5 publicaciones, la University of Manchester con 12 publicaciones, el Department of Geography and Environmental Development de la Ben Gurion University con 2 publicaciones, el Department of Geography de la Hong Kong University con 5 publicaciones, la University of Durham con 2 publicaciones, la School of Environment and Development de la University of Manchester con 1 publicación, el Department of Geography and Human Environment de la Tel Aviv University con 4 publicaciones y el Department of International Relations, de la London School of Economics and Political Science con 4 publicaciones.

\section{Area temática: Gobierno abierto}

Al realizar la búsqueda en Scopus Elsevier tomando en consideración la ecuación de búsqueda mencionada en la metodología de este artículo, se generaron 51 resultados de búsqueda entre los cuales se publica un $63 \%$ en formato artículo científico, un $23 \%$ en papers de conferencia, un $5 \%$ de capítulos de libro, un $2 \%$ en libros y un $2 \%$ en revisiones. Es decir, la comunidad de investigación, al publicar acerca del área temática "Instrumentos de medición de la transparencia y participación en el sector público", mayoritariamente utiliza los medios de publicación de artículos científicos, papers de conferencia, capítulos de libros, libros y revisiones.

La trayectoria de las publicaciones mencionadas y registradas en Scopus en esta área de estudio muestra que la primera publicación realizada en la materia fue durante el año 2013, lo cual evidencia que la línea de investigación es un campo emergente. A continuación, se detalla esta producción científica (Gráfico 9). 


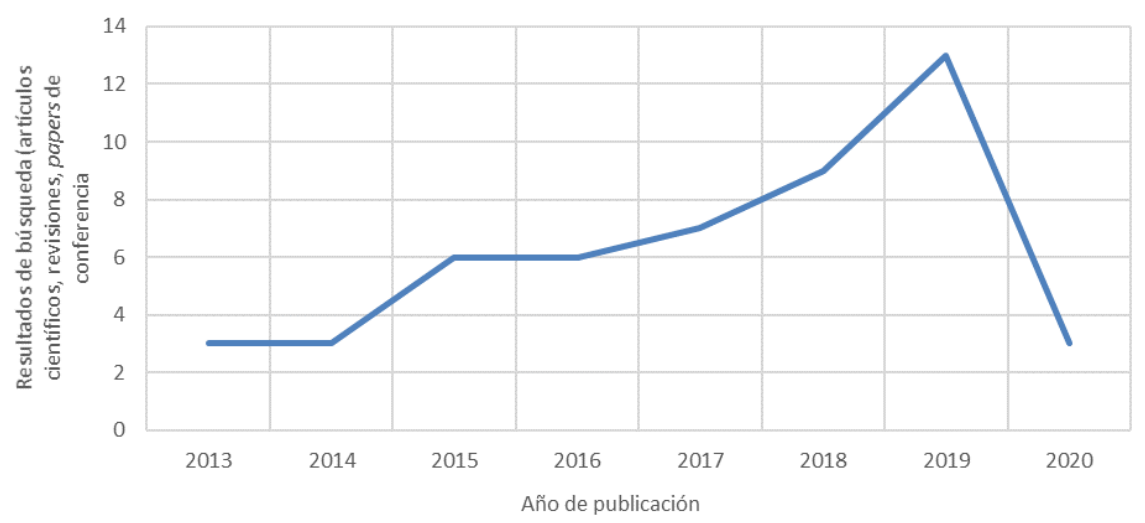

Gráfico 9.

Trayectoria de la producción científica. Línea de investigación: Instrumentos de medición de la transparencia y participación en el sector público (2000 - 2020)* *No hay publicaciones registradas en Scopus antes del 2013. Fuente: elaboración propia.

A diferencia de las dos líneas de investigación presentadas, en este apartado se inicia en años más tempranos, lo cual hace pensar en lo reciente del campo del conocimiento sobre las mediciones de la transparencia y la participación en el sector público. Asimismo en el gráfico anterior, se muestra que las publicaciones anuales en este campo tienden a crecer año tras año, lo cual podría ser una referencia del interés que tiene la comunidad de investigación en publicar en esta área temática.

En cuanto a la cantidad de publicaciones por personas autoras, los resultados arrojan que existen autores cuya cantidad de publicaciones no destaca por sobre otros autores, siendo que del top 10 de autores que más han publicado en la materia 8 de ellos han publicado 2 artículos científicos, estos autores son los siguientes: Dahbi, K., Hackett, R.A., Lamharhar, H., Lesage, F., Lourenço, R., Morandini, L. y Sinnott, R. Adicionalmente, existen 35 autores que han publicado un artículo científico en esta área temática.

$\mathrm{Al}$ igual que en las dos líneas de investigación anteriores, las personas autoras en publican mayoritariamente en el idioma inglés. Sin embargo, se destaca que además de publicar en revistas, también se publica en conferencias. Por lo tanto, el top 10 de los medios de en los cuales se publica son los siguientes: en cuanto a conferencias, en la con 4 publicaciones, $\mathrm{L}$ con 2 publicaciones y la Comparing The Democratic Governance of Police Intelligence New Models of Participation And Expertise In The United States and Europe con 1 publicación. En cuanto a la publicación en revistas, la Tabla 3 destaca las top 10. 
Tabla 3.

Top 10 de los medios que se publica más acerca de la línea de investigación "Instrumentos de medición de la transparencia y participación en el sector público” (2000 - 2020)

\begin{tabular}{|c|c|}
\hline Nombre de la revista & $\begin{array}{l}\text { Número de } \\
\text { artículos } \\
\text { publicados }\end{array}$ \\
\hline $\begin{array}{l}\text { ACM International Conference Proceeding } \\
\text { Series }\end{array}$ & 4 \\
\hline $\begin{array}{l}\text { Lecture Notes In Computer Science Including } \\
\text { Subseries Lecture Notes In Artificial } \\
\text { Intelligence And Lecture Notes In } \\
\text { Bioinformatics }\end{array}$ & 2 \\
\hline Media and Communication & 2 \\
\hline $\begin{array}{l}\text { American Behavioral Scientist con una } \\
\text { publicación }\end{array}$ & 1 \\
\hline $\begin{array}{l}\text { Between The Public and Private in Mobile } \\
\text { Communication }\end{array}$ & 1 \\
\hline Big Data And Society & 1 \\
\hline Campbell Systematic Reviews & 1 \\
\hline Ceur Workshop Proceedings & 1 \\
\hline Communication And Society & 1 \\
\hline
\end{tabular}

Fuente: elaboración propia

En cuanto a las instituciones académicas que más publican en la materia, y que se encuentran en el top 10, se encuentran las siguientes: con 4 publicaciones destaca la Universidade de Coimbra; con 2 publicaciones destacan el King's College Londo, la University at Albany, Simon Fraser University, Mohammed $\mathrm{V}$ University in Rabat, University of Twente, University of Melbourne; y finalmente con una publicación destacan el National Informatics Centre y el Shodor Education Foundation Inc.

\section{Área temática: Administración Aduanera y Comercio Exterior}

$\mathrm{Al}$ realizar la búsqueda en la base de datos, se generaron 80 resultados, entre los cuales se publica un $49 \%$ en formato artículo científico, un 30\% en papers de conferencia, un $8 \%$ de capítulos de libro, un $6 \%$ en revisiones, un $4 \%$ en libros. Es decir, al igual que en el área temática de Gobierno Abierto, destacan los artículos científicos y lospapers de conferencia como los medios de mayor uso para publicar en esta área temática, y mucho menor medida capítulos de libros, revisiones y libros.

La trayectoria de las publicaciones (Gráfico 9) muestra un comportamiento desordenado de la cantidad de publicaciones, aunque se denote una tendencia de crecimiento al largo de los años. 


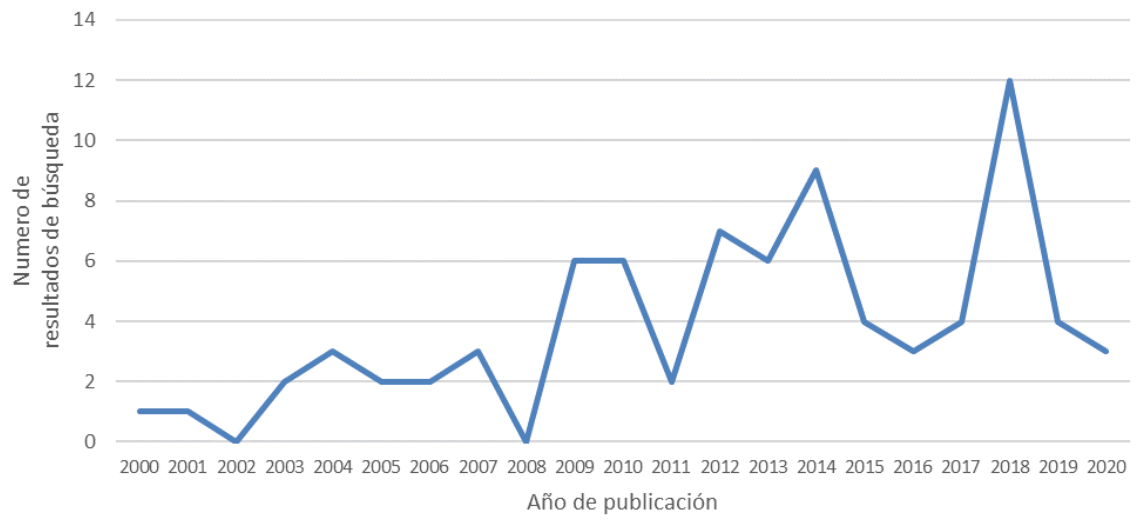

Gráfico 9.

Trayectoria de la producción científica. Línea de investigación: Sistemas informáticos aduaneros y facilitación del comercio (2000-2020). Fuente: elaboración propia.

En cuanto a la cantidad de publicaciones por personas autoras, los resultados arrojan que existen 95 autores que han publicado un resultado científico entre artículos, papers de conferencias, libros, capítulos de libros y revisiones. Por lo que no se encontró autores que sobresalieran en su cantidad de artículos publicados.

$\mathrm{Al}$ igual que en las líneas de investigación anteriores, las personas autoras en esta publican mayoritariamente en el idioma inglés. Sin embargo, se destaca que además de publicar en revistas, también se publica en conferencias, por lo tanto, el top 10 de los medios en los que se publica son los siguientes (Tabla 4):

\section{Tabla 4 .}

Top 10 de los medios que se publica más acerca de la línea de investigación "Sistemas informáticos aduaneros y facilitación del comercio" (2000 - 2020)

\begin{tabular}{|ll|}
\hline Nombre de la revista & $\begin{array}{l}\text { Número de } \\
\text { artículos } \\
\text { publicados }\end{array}$ \\
\hline $\begin{array}{l}\text { Lecture Notes In Computer Science Including } \\
\text { Subseries Lecture Notes In Artificial Intelligence And } \\
\text { Lecture Notes In Bioinformatics }\end{array}$ & 2 \\
17 th European Conference On Information Systems & 1 \\
Ecis 2009 & \\
2010 International Conference On Intelligent & 1 \\
Computation Technology And Automation Icicta & \\
2010 & \\
2012 IEEE SICE International Symposium On & 1 \\
System Integration Sii 2012 & 1 \\
2013 IEEE Photonics Conference IPC 2013 & 1 \\
2014 37th International Convention On \\
Information And Communication Technology \\
Electronics And Microelectronics Mipro 2014 \\
$\begin{array}{l}\text { Proceedings } \\
\text { 2018 22nd International Computer Science And } \\
\text { Engineering Conference Icsec }\end{array}$ \\
$\begin{array}{l}\text { World Customs Journal } \\
\text { Global Trade And Customs Journalcon } \\
\text { SAE Technical Papers }\end{array}$ & 1 \\
\hline
\end{tabular}

Fuente: elaboración propia 
En cuanto a las instituciones académicas que más publican en la materia, se encuentran las siguientes: Aarhus Universitet con 2 publicaciones; y con una publicación se destacan General Motors Do Brasil, Crowder Consulting, Hypo Real Estate Holding AG, Nine of July University UNINOV, Commanding Communications Academy, National Museum of Denmark, Micro Photon Devices y Southern African Development Community SADC.

\section{Discusión}

En el presente estudio se parte de la producción científica de los últimos 20 años en el CICAP (2000-2020), considerándola como un insumo para comprender las áreas temáticas en las que el Centro ha priorizado. Por medio de análisis bibliométrico y de entrevistas realizadas a las personas investigadoras, se confirma que el CICAP ha pasado por dos momentos en su trayectoria de investigación: antes del 2010, por periodo caracterizado por la organización de congresos, seminarios y foros; y después del 2010, por un período en donde las publicaciones fueron el mayor resultado de investigación. Lo anterior es bastante relevante para comprender el campo de conocimiento y su desarrollo en un Centro de la Administración Pública de relevancia nacional.

Para tomar decisiones basadas en evidencia en las cuatro líneas de investigación prioritarias a fin de integrar el acervo de conocimiento mundial con el realizado por el CICAP, esta trayectoria no debe aislarse de la producción científica mundial en la materia. Lo anterior se torna relevante al identificar en el estudio que uno de los temas del campo de la Administración Pública identificado por la Plataforma Scival con un indicador de prominencia alto fue el de "Gobierno Abierto", mismo tema en el que el CICAP ha emergido con la producción anual de instrumentos de medición de la transparencia y participación en el sector público por medio del cálculo del Indice de Transparencia en el Sector Público Costarricense, pero que a la fecha no ha capitalizado este esfuerzo en publicaciones de artículos científicos en revistas indexadas.

Es por esta razón que la realización de este estudio discute a partir de acciones efectivas y sistemáticas para que en Centros de Investigación, y en particular en el CICAP, se generen productos de investigación que aporten al crecimiento y maduración de la investigación en Administración Pública, estando en concordancia con lo que expone Fadul (2011) acerca de la necesidad de iniciativas no aisladas de análisis de la producción científica, para buscar la construcción de conocimientos científicos acumulados que contribuyan en el desarrollo de este campo de estudio. La administración pública necesita un análisis bibliométrico para profundizar la comprensión de la evolución del campo a lo largo del tiempo (Chandra \& Walker, 2019).

En este artículo se evidencia que el campo de estudio de la Administración Pública, tanto en el CICAP, así como en las cuatro líneas de investigación priorizadas para el estudio bibliométrico, se realizan contribuciones científicas cuyo comportamiento tiende a la alza en los formatos de artículos científicos, capítulos de libros y libros, pudiendo ser, según Guerrero-Orozco (2014), una de las disciplinas de las Ciencias Sociales que más ha producido literatura en el campo de la investigación aplicada. Por consiguiente, la comprensión de la dinámica y evolución de temas de interés en la comunidad científica es 
una de las aportaciones de los estudios bibliométricos (Smolski et al., 2017), ya que "sintetizar investigaciones previas o 'hacer balance del campo' ha sido considerado uno de los caminos más importantes para el avance del conocimiento en cualquier disciplina" (Chandra \& Walker, 2019, p. 716, traducción propia).

Los resultados muestran que la Administración Pública esta evolucionando y consolidándose, desde los estudios de Mezzomo y Laporta (1994) y Fleury y colaboradores (2003); viene modificándose en cuanto al perfil de los autores, bases teóricas utilizadas y territorios explorados. La investigación realizada por el CICAP, especialmente por la vocación dirigida a la organización de congresos, seminarios, foros y simposios en el período 2000-2010, y la vocación de publicación de artículos científicos en los años subsecuentes, ha suscitado discusiones en el campo, mayoritariamente en áreas temáticas como Administración Pública en general, Desarrollo Municipal, Gobierno Abierto y Administración Aduanera.

La combinación de indicadores bibliométricos con datos cualitativos desde las entrevistas con personas investigadoras permitió profundizar en estas líneas de investigación, destacando, en este sentido, la Administración Pública en el desarrollo de la gestión del territorio en el marco del capitalismo, la gestión del territorio y territorialidad; instrumentos para medir la transparencia y la participación del sector público, y sistemas informáticos aduaneros y facilitación del comercio.

Esta comprensión más profunda de las áreas temáticas y sus líneas de investigación reveló lagunas de investigación que pueden orientar la dirección de las personas investigadoras en su análisis futuro, tanto en toda el área de la Administración Pública como en el propio CICAP. Puede contribuir también a la práctica de la gestión pública, como lo han hecho estudios como el de Chandra \& Walker (2019), cuya contribución destaca la influencia de un artículo seminal sobre la estructura intelectual del campo de la administración pública, y de Zeemering (2018), que muestra cómo el estudio de una nación puede influir en el diálogo global sobre gestión pública y beneficiar la práctica de gobernar.

Nuestro estudio también indica que el comportamiento de la producción científica indexada en la base de datos Scopus Elsevier en las líneas de investigación priorizadas por la CICAP exige la participación de países e instituciones de las economías periféricas, incluida la República de Costa Rica, desde el predominio de Estados Unidos de Norteamérica-Europa en la corriente principal de la discusión. De este modo, la producción científica realizada en el CICAP necesita difusión a nivel internacional tanto en revistas internacionales indexadas especializadas en Administración Pública, así como en congresos internacionales reconocidos en la materia, para producir un mayor impacto científico en las investigaciones en el campo.

Para finalizar, no se debe obviar el alcance teórico y metodológico de la bibliometría como técnica para tomar decisiones basadas en evidencia, ya que sus análisis se circunscriben a la generación de métricas que muestran impactos científicos (Vonortas 2017), es decir en el acervo del conocimiento y en las comunidades científicas, lo cual puede ser complementado con métricas alternativas que midan impactos sociales de la producción científica en redes sociales, en políticas públicas y en la opinión pública, como las métricas expuestas por Priem et. al. (2010). 


\section{Referencias}

Aguilera Gámez, I. A. (2020). Transformación digital en la administración pública Un estudio bibliométrico.

Araújo, C. A. (2006) Bibliometria: evolução história e questões atuais. Em Questão, Porto Alegre, v. 12, n. 1, p. 11-32, jan./jun. 2006.

Bin, A. (2008). Planejamento e gestão da pesquisa e da inovação: conceitos e instrumentos. Tese de doutorado em Política Científica e Tecnológica, Universidade Estadual de Campinas, Unicamp, Campinas, SP.

Benavente, J. M., Crespi, G., Garone, L. F., \& Maffioli, A. (2012). The impact of national research funds: A regression discontinuity approach to the Chilean FONDECYT. Research Policy, 41(8), 1461-1475.

Chandra, Y., \& Walker, R. M. (2019). How does a seminal article in public administration diffuse and influence the field? Bibliometric methods and the case of Hood's "A Public Management For All Seasons?". International Public Management Journal, 22(5), 712-742.

Chueke, G. V., \& Amatucci, M. (2015). O que é bibliometria? Uma introdução ao Fórum. Internext, 10(2), 1-5.

CICAP. (2020). Informe de Resultados del Índice de Transparencia del Sector Público, 2020. Centro de investigación y Capacitación en Administración Pública de la Universidad de Costa Rica.

CICAP. (2019). Informe de Resultados del Índice de Transparencia del Sector Público, 2020. Centro de investigación y Capacitación en Administración Pública de la Universidad de Costa Rica.

CICAP. (2018). Informe de Resultados del Índice de Transparencia del Sector Público, 2020. Centro de investigación y Capacitación en Administración Pública de la Universidad de Costa Rica.

CICAP. (2017). Informe de Resultados del Índice de Transparencia del Sector Público, 2020. Centro de investigación y Capacitación en Administración Pública de la Universidad de Costa Rica.

CICAP. (2016). Informe de Resultados del Índice de Transparencia del Sector Público, 2020. Centro de investigación y Capacitación en Administración Pública de la Universidad de Costa Rica.

CICAP. (2015). Informe de Resultados del Índice de Transparencia del Sector Público, 2020. Centro de investigación y Capacitación en Administración Pública de la Universidad de Costa Rica.

Cicea, C. (2020). Performance in public management. A research agenda with bibliometric approach. Administratie si Management Public, 35, 109-123. DOI: $10.24818 / \mathrm{amp} / 2020.35-07$

Cozzens, S.E. (2012). Frontiers of evaluation: United States. Revista Brasileira de Inovação, Campinas (SP), 11(n. esp.), p. 47-66.

Dias, M. A. H., Zarelli, P. R., \& Selig, P. M. (2014). Intellectual capital and public management: a bibliometric analysis. Journal of US-China Public Administration, 11(2), 108-120.

Daim, T.U., Rueda, G., Martin, H. \&Gerdsri, P. (2006). Forecasting emerging technologies: Use of bibliometrics and patent analysis. Technological Forecasting and Social Change, 73(8), 981-1012.

Freitas, J. L., Bufrem, L. S., de Oliveira, E. F. T., \& Gracio, M. C. C. (2017). El interdominio de los estudios métricos de la información en Iberoamérica y 
Sudáfrica: análisis en la base SciELO en el período 1978-2013. Revista Cubana de Información en Ciencias de la Salud, 28(1), 1-1.

Fadul, Élvia; Silva, Lindomar P.; Cerqueira, Lucas S. (2011). Uma análise do campo da administração pública através da produção científica publicada nos Anais dos EnAPGs. Cadernos Gestão Pública e Cidadania, São Paulo, v. 16, n. 59.

Furtado, A.T.; Bin, A.; Bonacelli, M.B.M.; Paulino, S.R.; Miglino, M.A.; Castro, P.F.D. (2009). Evaluation of the results and impacts of a social-oriented technology program In Brazil: the case of Prosab (a sanitation research program). Research Evaluation, 18, 2009.p. 289 - 300

Hocayen-da-Silva, A. J., Rossoni, L., \& Ferreira Júnior, I. (2008). Public sector management and social management: brazilian scientific production between 2000 and 2005. Revista de Administração Pública, 42(4), 655-680.

Juliani, F., \& de Oliveira, O. J. (2016). State of research on public service management: Identifying scientific gaps from a bibliometric study. International Journal of Information Management, 36(6), 1033-1041.

Lin, P. H., Chen, J. R., \& Yang, C. H. (2014). Academic research resources and academic quality: a cross-country analysis. Scientometrics, 101(1), 109-123.

ELSEVIER. Topic Prominence in Science FAQs (2020). Disponível em: $<$ https://serv ice.elsevier.com/app/answers/detail/a_id/28428/supporthub/scival/>. . Acesso em: 26 mar. 2020.

MICITT (2019). Indicadores Nacional de Ciencia, Tecnología e Innovación de Costa Rica 2018.

Menandro, P. R. M. (2003). Linha de pesquisa: possibilidades de definição e tipos de utilização do conceito. Revista de Administração Contemporânea, 7(2), 177-182

Moya, A. (2012). CICAP MEMORIA XXXV ANIVERSARIO 1976-2011. La senda del éxito y la superación constante. Universidad de Costa Rica.

Mongeon, P., \& Paul-Hus, A. (2016). The journal coverage of Web of Science and Scopus: a comparative analysis. Scientometrics, 106(1), 213-228.

Pérez, J. P. M. (2007). 25 años de la Revista Centroamericana de Administración Pública del ICAP: Indicadores bibliométricos 1981-2006. Revista Centroamericana de Administración Pública, (52-53).

Price, D. J. S. (1963). Little science, big science. New York: Columbia University Press.

Priem, D. Taraborelli, P. Groth, C. Neylon (2010), Altmetrics: A manifesto, October 2010.

Vonortas, L. (2017). Bibliometrics. Session 9 of the course Impact Evaluation of R\&D and Innovation. Executive Course. C Center for Int'l Science and Technology Policy and Department of Economics - The George Washington University Sao Paulo. Departamento de Política Científica e Tecnológica - Universidade Estadual de Campinas (UNICAMP).

Spinak, E. (1996). Diccionario enciclopédico de Bibliometría, Cienciometria e Infometría. Caracas: UNESCO.

Smolski, Felipe Micail da Silva, Dalcin, Dionéia, Visentini, Monize Sâmara, Bamberg, Joice, \& Kern, Juliana Strieder. (2017). Análise do perfil da produção científica da Revista de Administração Pública (RAP) no período 2003-16. Revista de Administração Pública, 51(6), 1139-1163. https://doi.org/10.1590/0034-76122 0170046

Zeemering, E. S. (2018). The global relevance of Canadian public administration? A bibliometric research note. Canadian Public Administration, 61(3), 385-406. 
Notas

1 Un tema (topic) es una colección de documentos con un enfoque específico de interés intelectual (ELSEVIER, 2020). Es decir, es una agrupación de publicaciones organizadas en torno a un núcleo común, que puede ser un tema, un área de conocimiento, un método o otros criterios. Metodológicamente, las publicaciones de Scopus se agrupan bajo estos temas en base a un análisis de citas directo. La plataforma considera toda la red de citas (más de mil millones de enlaces entre más de 48 millones de documentos indexados en Scopus desde 1996 y más de 20 millones de documentos no indexados citados al menos dos veces) y divide esta red en aproximadamente 96,000 temas.

2 Para indicar el "tiempo" de un tema, se combinan tres métricas: (i) recuento de citas en el año "n" para artículos publicados en "n" y "n-1"; (ii) recuento de vistas (vistas) en el año "n" para artículos publicados en n y n-1; (iii) CiteScore promedio para el año "n".

3 Cabe aclarar que la producción científica relativa al año 2020 puede parcialmente ser explicada por el echo de ser un año incompleto en las bases científicas e, por tanto, pueden surgir más publicaciones al terminar el año.

4 Las áreas temáticas son las siguientes: Servicios públicos, Recursos humanos, Desarrollo Municipal, Administración aduanera, Finanzas, Gestión de la ciencia y la tecnología, Gerencia, Contabilidad y control gerencial, Gobierno Abierto, Reforma administrativa, Monitoreo y Evaluación, Gobierno Digital, Contratación Administrativa, Estudios organizacionales, Estrategia y prospectiva, Economía social y solidaria, Política social, Mercadeo, Gestión de la ciencia y la tecnología, Gestión del recurso hídrico, Política de salud, Gestión del riesgo, Planificación gubernamental, Ciencia de datos y Economía social y solidaria. 